\title{
Positive pulse switching in coupled nonlinear Fabry-Perot cavities
}

\author{
Kwongchoi Caisy Ho and Guy Indebetouw
}

\begin{abstract}
It is shown that positive pulse switching is possible in a system of two coupled nonlinear Fabry-Perot cavities. The conditions in which a signal beam can be switched on and off by positive pulses in the steady state regime are described for cavities filled with Kerr media having a long diffusion length.
\end{abstract}

\section{Introduction}

Nonlinear Fabry-Perot cavities, in particular the interference filters and semiconductor etalons, have been extensively studied in the recent past because of their potential as simple and versatile all optical bistable elements. ${ }^{1,2}$ Conventionally, a bistable FabryPerot cavity is operated with a constant bias. A pulse is added to the bias to switch the device from an off state of low transmittance to an on state of higher transmittance. Down switching is achieved by interrupting the bias, i.e., by adding a negative pulse to it. As this interruption is dissipative and thus, in principle, not conducive to very high speed, ${ }^{3}$ the search for systems in which both on and off switching are triggered by positive pulses is of great interest. Also, the requirement for more than two signal levels (positive, negative, and bias) is not compatible with conventional digital circuitry where gates are driven by pulses having a single polarity.

Positive pulse switching does not appear to be achievable in a conventional single input nonlinear cavity, at least not in the steady state regime. Even if the cavity is birefringent and polarization is being used as an additional design parameter, up switching in such systems is generally irreversible in the sense that the device eventually finds itself locked in the on state. ${ }^{4}$ It is, however, possible to down switch a cavity with a positive pulse if two different mechanisms are responsible for the up and the down switching. Down

The authors are with Virginia Polytechnic Institute \& State University, Physics Department, Blacksburg, Virginia 24061-0435.

Received 11 July 1990.

0003-6935/91/182437-06\$05.00/0.

(C) 1991 Optical Society of America. switching then results from a momentary shift of the hysteresis loop through the bias point.

Devices utilizing the thermal effect in semiconductors ${ }^{5}$ or the transient shift of the transmission peaks of an etalon ${ }^{6}$ have been demonstrated. The shift of the hysteresis loop can also be triggerd by the beats between the bias and a pulse of a different frequency. ${ }^{7}$ Another broad class of devices in which positive pulse switching is in principle achievable includes the coupled cavities systems. ${ }^{8}$ This possibility has been demonstrated in systems coupled by external feedback loops, ${ }^{9}$ in coupled lasers, ${ }^{10}$ and more recently in dual input cavities in which the bistability results from a pitchfork bifurcation ${ }^{11,12}$ as well as in symmetric selfelectrooptic effect devices. ${ }^{13}$

The aim of this paper is to show that positive pulse switching is achievable in two optically coupled nonlinear Fabry-Perot cavities in the steady state regime. The coupled cavities can be either two separate cavities or two nonoverlapping elements of the same cavity. The model used to describe the cavities and its underlying assumptions are outlined in the next section. The simplest possible model is chosen, and the analysis is limited to the steady state regime. The response of the device is described in Sec. III and demonstrated with numerical examples. Finally, some of the limitations and requirements for positive pulse switching in the steady state regime are discussed. The motivation for this analysis is twofold. Positive pulse switching is conceptually important for the reasons already mentioned. Thus, defining the types of system in which it is achievable is of definite interest. Furthermore, coupling between cavities arranged in series or between different cavity elements can occur either intentionally or accidentally in cascaded devices, which are of great practical importance.

\section{Theoretical Model}

Figure 1 shows schematically the geometry of the systems considered. It consists of two nonlinear 


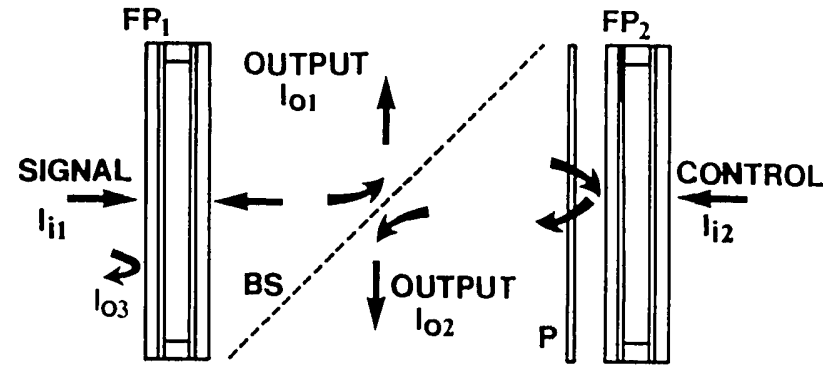

Fig. 1. Schematic diagram of the coupled nonlinear cavities $F P_{1}$, $F P_{2}$, Fabry-Perot cavities filled with Kerr-type nonlinear media with long diffusion length: $I_{i 1}$, signal input; $I_{i 2}$, control input; $B S$, beam splitter; and $P$, polarizer transmitting $I_{i 1}$, but not $I_{i 2}, I_{o 1}, I_{o 2}, I_{o 3}$, possible outputs.

Fabry-Perot cavities $F P_{1}$ and $F P_{2}$ arranged in series. Each cavity is made of two dielectric lossless mirrors of power reflectivity $R_{1}$ for $F P_{1}$ and $R_{2}$ for $F P_{2}$. The cavities are filled with homogeneous Kerr-type media having a long diffusion length. As a result, the cavities are characterized by conventional hystereses. Polarizer $P$ is used to isolate $F P_{1}$ from the control beam. The outputs which derive only from the signal beam are extracted by beam splitter $B S$.

The round trip phase change in each cavity depends only on the average intensity $I_{1}$ or $I_{2}$ of the radiation trapped in the cavity. The round trip phase change in cavity $j=1,2$ can thus be written as

$$
\phi_{j}=2 k d_{j}\left(n_{0 j}+n_{2 j} I_{j}\right) \text {, }
$$

where $k=2 \pi / \lambda$ is the wavenumber in vacuo, $\lambda$ is the wavelength, $n_{0 j}$ is the linear index of refraction of the medium in cavity $j$, and $n_{2 j}$ is the value of its nonlinear index.

There are two inputs to the system. Input $I_{i 1}$ on $F P_{1}$ is called the signal beam, while input $I_{i 2}$ on $F P_{2}$ is called the control beam. They have orthogonal polarization. Three outputs are also shown. Because the polarization isolates $F P_{1}$ from $I_{i 2}$, the outputs derive entirely from signal $I_{i 1}: I_{o 1}$ is the signal beam transmitted by $F P_{1}, I_{o 2}$ has experienced an additional reflection on $F P_{2}$, and $I_{o 3}$ is the signal beam reflected by $F P_{1}$. In the schematic diagram of Fig. 1, outputs $I_{o 1}$ and $I_{o 2}$ are extracted by beam splitter $B S$ placed between the two cavities, but other folded geometries are also possible.

Isolating cavity $F P_{1}$ from the control beam results in the simplest possible kind of coupling between the two cavities. The control beam does not reach cavity one and thus does not affect it directly. The important point is that the control beam can change the reflectivity of $F P_{2}$, which affects the total power incident on $F P_{1}$ and thus changes the shapes of its hysteretic response curve.

It is further assumed that the two cavities are coupled incoherently. That is, the irradiance reflected on $F P_{2}$ is fed back onto $F P_{1}$, changing the total irradiance inside that cavity, but the reflected radiation does not interfere with the incident signal beam. This situation is true when the coherence time of the radiation is shorter than the transit time between cavities or if the spacing between the cavities does not form a resonant cavity because of misalignment or other mistuning. This contrasts with other double-cavity systems previously considered. ${ }^{14}$

For each cavity, there are four time scales in the system: The cavity round trip times $\tau_{R_{j}}$, the cavity lifetimes $\tau_{C_{j}}$, the medium response times $\tau_{M_{j}}$, and the time scale of input changes $\tau_{P_{j}}$ (i.e., the pulses rise and fall times). An additional time scale is the transit time $\tau_{T}$ between the cavities. The steady state solution is valid when $\tau_{T}, \tau_{R_{j}}<\tau_{C_{j}} \ll \tau_{M_{j}} \ll \tau_{P_{j}}$; here $j=1,2$.

With these assumptions and using a plane wave approach, the average irradiance inside each cavity can be calculated by summing up the contributions after each round trip, giving the result

$$
\begin{aligned}
& I_{1}=\frac{1+R_{1}}{1-R_{1}} \mathcal{T}_{1} I_{i_{1}}\left(1+\frac{T^{2} \tau_{1} \mathcal{R}_{1}}{1-T^{2} \mathcal{R}_{1} \mathcal{R}_{2}}\right), \\
& I_{2}=\frac{1+R_{2}}{1-R_{2}}\left(\mathcal{T}_{1} I_{i_{2}}+\frac{T^{2} \tau_{1} \mathcal{T}_{2}}{1-T^{2} \mathcal{R}_{1} \mathcal{R}_{2}} I_{i_{1}}\right) .
\end{aligned}
$$

In these expressions, the overall power transmittance of each cavity is given by the usual Airy function:

$$
\tau_{j}=\left(1-F_{j} \sin ^{2} \phi_{j} / 2\right)^{-1} \text {; here } j=1,2,
$$

and the overall reflectance $\mathcal{R}_{j}=1-\mathcal{T}_{j}$ for lossless cavities. Term $F_{j}$ is the finesse of cavity $j$, the round trip phase change $\phi_{j}$ is given by Eq. (1), an $T$ is the transmittance of the beam splitter.

Equations (1)-(4) were solved numerically by searching for consistent solutions for the round trip phases $\phi_{j}$ in each cavity for all pairs of input values $I_{i 1}$ and $I_{i 2}$. The outputs were then calculated as

$$
\begin{aligned}
I_{o 1} & =\frac{R T_{1}}{1-T^{2} \mathscr{R}_{1} \mathcal{R}_{2}} I_{i 1}, \\
I_{\mathrm{o} 2} & =T \mathscr{R}_{2} I_{o 1},
\end{aligned}
$$

where $R$ is the reflectance of the beam splitter and $T$ is its transmittance.

\section{Numerical Results}

There are two possible modes of operation for the device of Fig. 1. In the first mode, the device is switched on and off by two control pulses of different magnitudes, while in the second mode it is switched on by a signal pulse and switched off by a control pulse.

In both cases, cavity $F P_{1}$ is biased within its bistable region with a constant input signal beam and with the reflection of that beam on cavity $F P_{2}$. The cavity $F P_{2}$ need not be biased and acts as a mirror of variable reflectance. The control pulses are used to change the reflectance of $F P_{2}$ and consequently switch the device.

\section{A. On and Off Switching with Control Pulses}

In the first mode of operation, cavity $F P_{2}$ must also be bistable. Control pulses of different magnitude are used to change the reflectance of $F P_{2}$. This changes the total bias of $F P_{1}$ and thus shifts the operating point on the hysteretic transfer function of the device, re- 


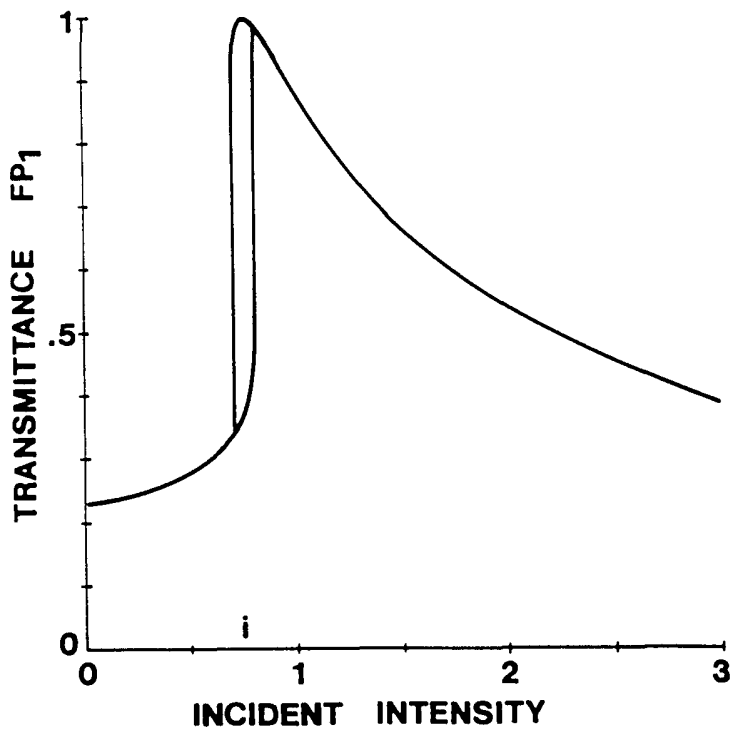

Fig. 2. Transmittance of $F P_{1}$ (uncoupled) with the following cavity parameters: $R_{1}=0.4$; initial detuning $\phi_{01}=2 k d_{1} n_{01}=1.50 \mathrm{rd} \mathrm{mod}$ $2 \pi$; and nonlinear index $n_{21}=10^{-3}$ r.u.

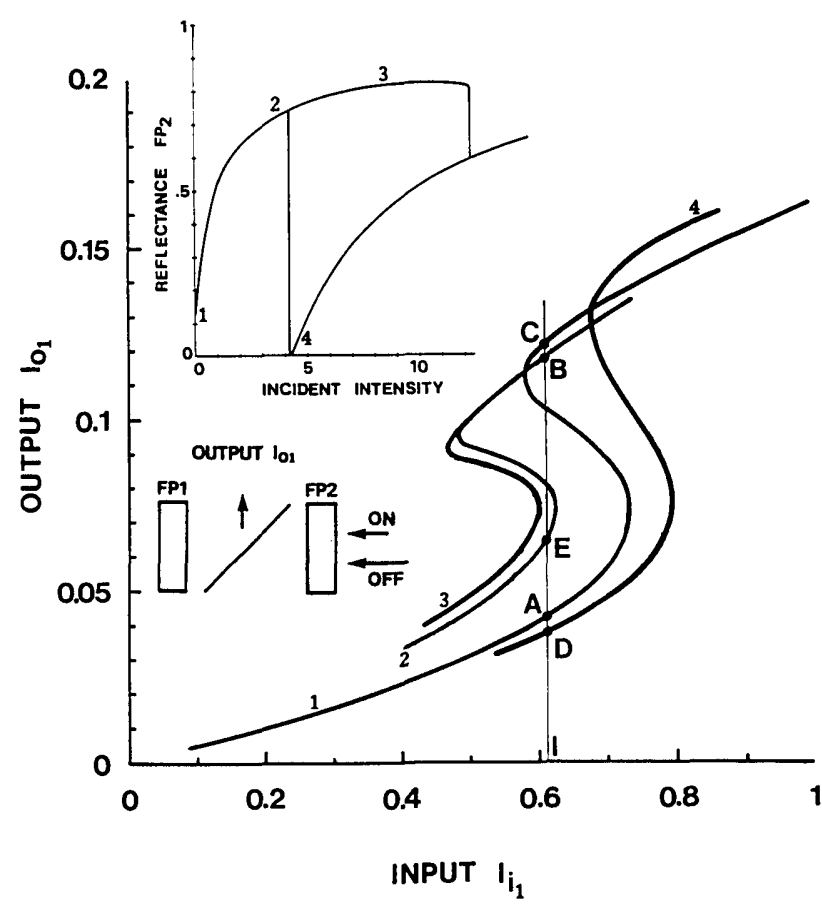

Fig. 3. Steady state output $I_{o 1}$ vs signal input $I_{i 1}$ for different values of the control beam intensity. Signal bias, $I$ : (1) output for $I_{i 2}=0$; (2) output for $I_{i 2}=3.4$; (3) output for $I_{i 2}=11$ corresponding to the upper branch of the hysteresis loop of $\mathcal{R}_{2}$; and (4) output for $I_{i 2}=4.2$ corresponding to the lower branch of the hysteresis loop of $\mathcal{R}_{2}$. The output is switched on $\left(A\right.$ to $B$ ) by control beam intensity $I_{i 2} \cong 11$. It relaxes to $C$ as the control pulse ends. The output is switched off by control beam intensity $I_{i 2}>11$. When the pulse ends, the output follows the lower branch of the hysteresis loop of $\mathcal{R}_{2}(C$ to $D$ ). Switch down occurs near point 4 (inset). The output shifts from $D$ to $E$ as $\mathcal{R}_{2}$ switches back up and finally relaxes back to $A$. For the output not to switch back on, $\mathrm{FP}_{1}$ must be biased between the switch-up points of curve 2 and 3.

Inset. Reflectance of $F P_{2}$ (uncoupled) with the following cavity parameters: $R_{1}=R_{2}=0.4$, initial detuning $\phi_{02}=3.94 r d \bmod 2 \pi$, and nonlinear index $n_{22}=5 \times 10^{-4}$ r.u.

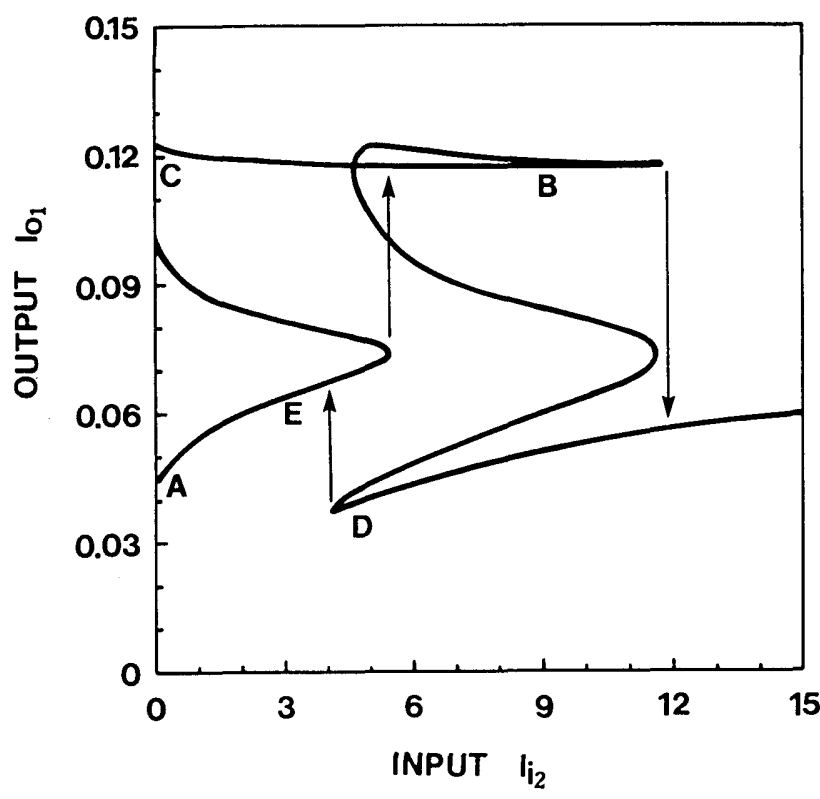

Fig. 4. Steady state output $I_{01}$ vs control signal input $I_{i 2}$. The output is switched on by a small control pulse $(A \rightarrow B \rightarrow C)$ and switched off by a larger control pulse $(C \rightarrow D \rightarrow E \rightarrow A)$.

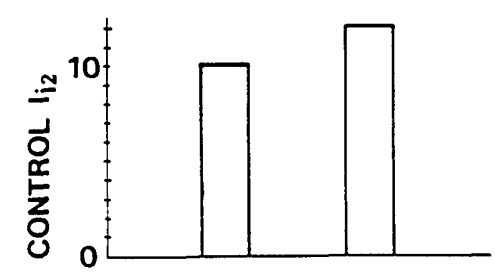

SIGNAL BIAS $l_{i 1}=$.

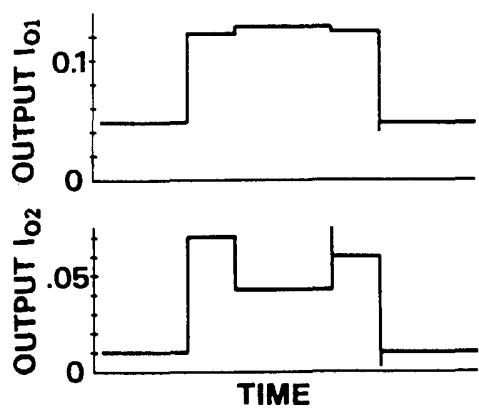

Fig. 5. Steady state outputs illustrating a switching sequence in which up and down switching are both triggered by control pulses of different magnitudes. The cavity parameters are the same as those for Figs. 2 and 3.

sulting in the device switching either on or off depending on the magnitude of the control beam.

The result of a numerical simulation is illustrated in Figs. 2-5. Transmittance $\mathcal{T}_{1}$ of $F P_{1}$ and the reflectance $\mathscr{R}_{2}$ of $F P_{2}$ are shown in Fig. 2 and in the inset of Fig. 3, respectively, as functions of the incident intensity on the respective uncoupled cavity. The parameters of each cavity are chosen so that both $F P_{1}$ and $F P_{2}$ are bistable. Namely, $R_{1}=R_{2}=0.4$, the initial detunings $\left(\phi_{0 j}=2 k d_{j} n_{0 j}\right)$ are $\phi_{01}=1.50 r d, \phi_{02}=3.94 r d \mathrm{mod}$ 
$2 \pi$, and the nonlinear indices are $n_{21}=10^{-3}, n_{22}=5 \times$ $10^{-4}$. With the normalized intensity units used, $n_{2} \cong$ $10^{-3}$ corresponds approximately to the nonlinear coefficient due to thermal effects in a typical nematic liquid crystal (e.g., MBBA). The output coupling beam splitter has a transmittance of $80 \%$.

As is evident from Eqs. (2) and (3), the reflectance of $\mathrm{FP}_{2}$, which is a function of the total irradiance inside that cavity, can be controlled by varying the intensity of the control beam. Since $F P_{1}$ is biased by two beams, the incident bias signal beam and the signal beam reflected by $\mathrm{FP}_{2}$, changing the reflectance of $\mathrm{FP}_{2}$ alters the shape of $F P_{1}$ 's hysteretic response. In particular, it changes the values of the critical intensities at which the outputs, $I_{o 1}, I_{o 2}$, or $I_{o 3}$ switch on or off. Figure 3 shows four hysteretic response curves for the output $I_{o 1}$ as a function of input signal intensity $I_{i 1}$. Each curve corresponds to a different value of control beam intensity $I_{i 2}$ and thus to different values of the reflectance of $\mathrm{FP}_{2}$, as indicated by the corresponding number in the inset of Fig. 3.

With the control beam intensity equal to zero, $I_{i 2}=$ 0 , the device's response is described by curve 1 in Fig. 3 . Input $I_{i 1}$ biases $F P_{1}$ within its bistable region, while the reflectivity $F P_{2}$ is at 1 in the inset.

A switching cycle may start with the device in a low state (e.g., at point $A$ in Fig. 3). A control pulse of relatively small amplitude increases reflectance $\mathscr{R}_{2}$ up to region 3 in the inset. This pulse must not be large enough to carry $\mathscr{R}_{2}$ beyond its bistable region. With an increased feedback from the reflection on $F P_{2}$, the critical switch-up intensity of $F P_{1}$ drops. This results in a shift of the output hysteresis loop toward lower values of $I_{i 1}$, as shown by curve 3 . Since $F P_{1}$ is still biased at $I$, the output switches to the upper branch of its hysteresis loop (point $B$ ) as soon as the critical intensity for up switching drops below the bias. When the control pulse ends, the output response curve returns to curve 1 , leaving the output in a high state (point $C$ ).

Down switching is triggered by a more intense control pulse which brings $\mathscr{R}_{2}$ beyond its bistable region. Reflectance $\mathcal{R}_{2}$ then switches to its lower branch. As the control pulse ends, reflectance $\mathcal{R}_{2}$ drops to low values along its lower branch. This reduces the bias of $F P_{1}$ and increases its critical switch-down intensity (curve 4). When this critical switch-down intensity exceeds bias $I$, the output cannot be maintained in the upper branch and it switches back to a low state (point $D)$. Eventually, as $\mathcal{R}_{2}$ returns to its original states, cavity $F_{2}$ will switch back up and its reflectance will increase momentarily. This increased bias on $F P_{1}$ must not be sufficient to switch the output back on. For this, it is necessary that $\mathcal{R}_{2}(2)<\mathcal{R}_{2}(3)$, and cavity $F P_{1}$ must be biased between the crtical up-switching intensity of curve 2 and that of curve 3.

Another way to visualize the switching sequence is to plot the output vs the control beam intensity. This is shown in Fig. 4 for output $I_{o 1}$ and the same cavity system. This figure is self-explanatory: a small control pulse $I_{\text {up }}<I_{i 2}<I_{\text {down }}\left(I_{\text {up }} \sim 5.4, I_{\text {down }} \sim 11.9\right)$

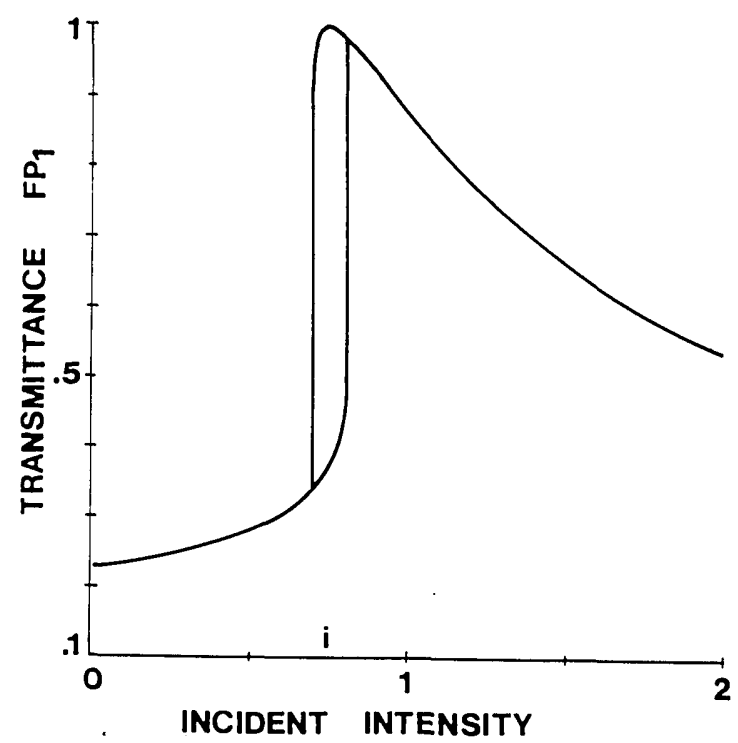

Fig. 6. Transmittance of $F P_{1}$ with the following cavity parameters: $R_{1}=0.4$, initial detuning $\phi_{01}=5.22 r d \bmod 2 \pi$, and nonlinear index $n_{21}=10^{-3}$ r.u.

switches the output up from $A$ to $B$ and to $C$ when the control pulse ends. A large control pulse $I_{i 2}>I_{\text {down }}$ switches the output down along the path $C$ to $D$ then to $E$ and eventually back to $A$ when the pulse ends.

Note that this figure also indicates that a constant bias $\left(I_{\text {bias }}<I^{\prime} \sim 4\right)$ could be used to maintain the control cavity closer to its switching point and thus to allow switching with smaller pulses.

The second output $I_{02}$ experiences an additional reflection on $\mathrm{FP}_{2}$. Consequently, the effect of the control pulses is not only to shift the hysteresis of the response curve but also to alter considerably the output intensity while the control pulses are present. Figure 5 illustrates the steady state switching sequence of the device just described.

\section{B. On and Off Switching With a Signal and a Control Pulse}

In this second mode of operation, the device is switched on by a signal pulse added incoherently to the bias signal in a manner similar to the switch-on of a single nonlinear cavity. It is then switched off by a control pulse in the same way as that described in the previous section.

The transmittance of $F P_{1}$ and the reflectance of $F P_{2}$, uncoupled, are shown in Fig. 6 and the inset of Fig. 7, respectively. The cavity parameters for this example are $R_{1}=R_{2}=0.4, \phi_{01}=\phi_{02}=5.22 r d \bmod 2 \pi, n_{21}=10^{-3}$, $n_{22}=5 \times 10^{-4}$. Note that with this initial detuning, $\mathscr{R}_{2}$ remains fairly constant on the upper branch of its hystersis. It would thus not be possible to switch on this device with a control pulse incident on $F P_{2}$.

Nonlinear cavity $F P_{1}$ is again biased within its bistable region. A trigger pulse added incoherently to the bias signal beam switches on the output from $A$ to $B$ in Fig. 7. This trigger pulse also increases the total input on $\mathrm{FP}_{2}$ and thus may reduce the reflectance of that cavity. This reduction, which acts as a negative 


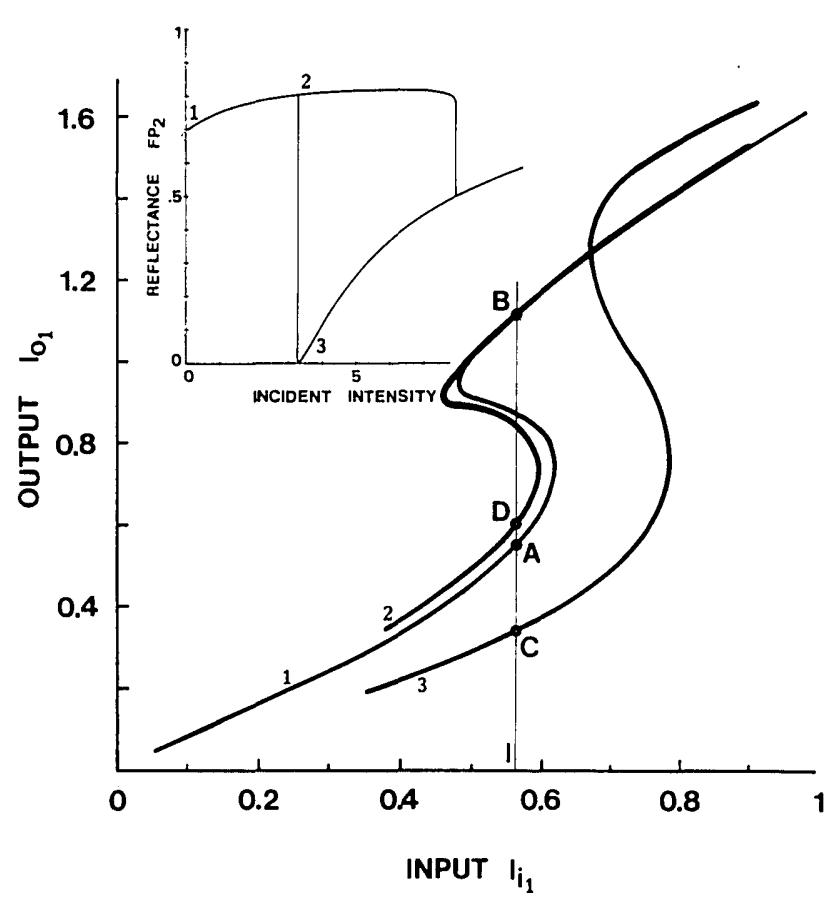

Fig. 7. Steady state output $I_{o 1}$ vs signal input $I_{i 1}$ for different values of the control beam intensity. Signal bias, $I$; (1) output for $I_{i 2}=0$; (2) and (3), output for $I_{i 2}=3.3$ corresponding to the upper and the lower branch of the hysteresis loop of $\mathcal{R}_{2}$. The output is switched on by a signal pulse $(A \rightarrow B)$ and switched off by a control pulse $(B \rightarrow C$ $\rightarrow D \rightarrow A$ ).

Inset. Reflectance of $F P_{2}$ with the following parameters: $R_{1}=R_{2}$ $=0.4, \phi_{02}=5.22 \mathrm{rd} \bmod 2 \pi$, and nonlinear index $n_{22}=5 \times 10^{-4}$ r.u.
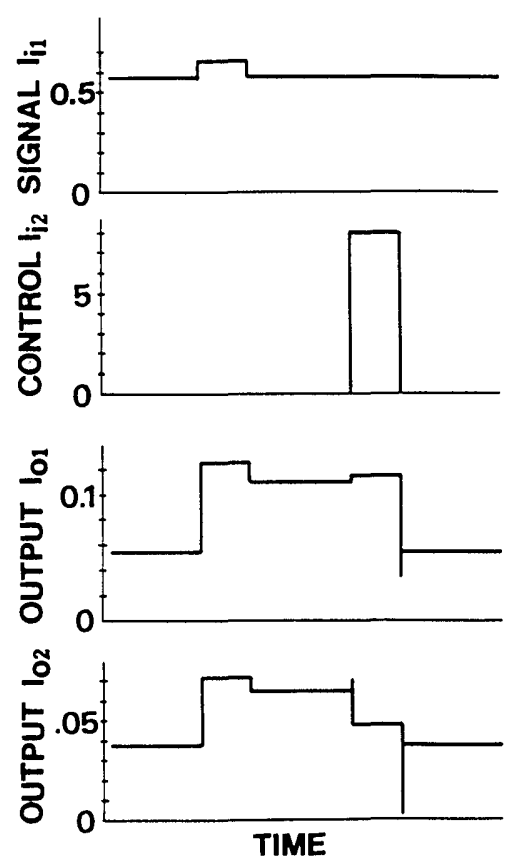

Fig. 8. Steady state outputs illustrating a switching sequence in which up switching is triggered by a signal pulse and down switching is triggered by a control pulse. feedback on $F P_{1}$, can be maintained small, however, by a proper choice of cavity parameters. As in the previous example, the output is switched back to a low state along the path $B C D A$ by a control pulse bringing $\mathcal{R}_{2}$ beyond its bistable region. The resulting steady state switching sequence of the device is shown in Fig. 8.

\section{Discussion and Conclusion}

We have demonstrated with numerical examples that positive pulse switching in the steady state regime is realizable in a system of two optically coupled nonlinear Fabry-Perot cavities filled with Kerr media having a long diffusion length.

Two modes of operation were described. In the first, a signal beam transmitted by the first cavity and extracted as an output is switched on and off by control pulses of different magnitudes incident on the second cavity. For this to be possible, both cavities must be bistable. Cavity one should be biased within its bistable region, and cavity two can be biased before its bistable region or can be left unbiased. Cavity one must also be biased in such a way that when $\mathcal{R}_{2}$ switches back up to a high reflectance, the output will remain in the lower branch of the hysteresis curve. Besides critical biasing requirements, this design needs two different amplitude levels to turn the device on and off, which is not compatible with binary logics. Another type of coupled cavity, which can be switched with pulses of equal magnitude, has been demonstrated experimentally. ${ }^{6}$ This device, which utilizes the finite recovery time of the cavity, requires two different wavelengths.

A second mode of operation of the system, in which the output is switched on by a signal pulse incident on one cavity and switched off by a control pulse incident on the other cavity was also described. The cavity parameter requirements are less critical for this device, since the control pulse must only shift the hysteresis loop in one direction by an amount just sufficient for the critical switch-down intensity to exceed the bias. The control cavity $F P_{2}$, for example, need not be bistable. An additional advantage of this design is that if $F P_{2}$ has a narrow bistable region, it can be biased close to its switching point. Consequently, only a small intensity control pulse is needed to switch the device off. Similarly, if $F P_{1}$ is biased close to its switching point, it can be switched on with a small intensity signal pulse. It is also possible, in principle, to adjust the parameters of the system to switch the device on and off with pulses of equal magnitude.

Part of the materials used in this paper was presented at the Annual Meeting of the Optical Society of America in Boston 1990.

\section{References}

1. H. M. Gibbs, Optical Bistability: Controlling Light Within Light (Academic, New York, 1985).

2. P. Mandel, S. D. Smith, and B. S. Wherrett, Eds., From Optical Bistability Toward Optical Computing (North-Holland, New York, 1987). 
3. A. Korpel and A. W. Lohmann, "Polarization and Optical Bistability," Appl. Opt. 25, 1528-1529 (1986).

4. K. C. Ho and G. Indebetouw, "Coupling of Orthogonal Polarization State in a Nonlinear Birefringent Cavity," Appl. Opt. 29, 206-209 (1990).

5. S.S. Tarng, K. Tai, J. L. Jewell, H. M. Gibbs, A. C. Gossard, S. L. McCall, A. Passner, T. N. C. Venkatesan, and W. Wiegmann, "External OFF and ON Switching of a Bistable Optical Device," Appl. Phys. Lett. 40, 205-207 (1982).

6. J. L. Jewell, M. C. Rushford, and H. M. Gibbs, "Use of a Single Nonlinear Fabry-Perot Etalon as Optical Logic Gates," Appl. Phys. Lett. 44, 172-174 (1983).

7. K. Inoue, "All Optical Flip-Flop Operation in an Optical Bistable Device Using Two Lights of Different Frequency," Opt. Lett. 12, 918-920 (1987).

8. A. Szoke, V. Daneu, J. Goldhar, and N. A. Kurnit, "Bistable Optical Element and Its Applications," Appl. Phys. Lett. 15, 376-379 (1969).
9. U. K. Sengupta, U. H. Gerlach, and S. A. Collins, "Bistable Optical Spatial Device Using Direct Optical Feedback," Opt. Lett. 3, 199-201 (1978).

10. J. L. Oudar and R. Kuszelewicz, "Bistabilite Optique des Lasers Couples,” Rev. Phys. Appl. 22, 1287-1295 (1987).

11. K. Otsuka "Pitchfork Bifurcation and All-Optical Digital Signal Processing with a Coupled-Element Bistable System," Opt. Lett. 14, 72-74 (1989).

12. M. Haelterman, P. Mandel, J. Danckaert, H. Thienport, and I. Veretennicoff, "Two-Beam Nonlinear Fabry-Perot Transmission Characteristics," Opt. Commun. 74, 238-244 (1989).

13. J. A. Goldstone and E. M. Garmire, "On the Dynamic Response of Nonlinear Fabry-Perot Interferometers," IEEE J. Quantum Electron QE-17, 366-374 (1981).

14. A. L. Lentine and H. S. Hinton, "Symmetric Self-Electro-Optic Effect Device: Optical Set-Reset Latch," Appl. Phys. Lett. 52, 1419-1421 (1988). 